\title{
Pathway Extension of Weibull-Gamma Model
}

\author{
T. Princy \\ Centre for Mathematical and Statistical Sciences \\ Peechi Campus, KFRI, Peechi-680653, Kerala, India \\ princycms@gmail.com
}

Received 17 March 2014

Accepted 18 November 2015

\begin{abstract}
In this paper we develop a new family of Weibull-gamma model which is obtained by compounding a $q$-Weibull density with a two parameter gamma density. This class can be considered as a natural extension of the Weibullgamma model which accommodates various other useful statistical models. Properties of the proposed model are discussed, including the derivation of its density function. The method of moments is used for estimating the model parameters. The practical importance of the new model is illustrated using cancer survival data.
\end{abstract}

Keywords: Meijer G-function; Mellin transform; H-function; compound models; Weibull distribution.

2000 Mathematics Subject Classification: 60E05, 44A10, 44A15, 33C60

\section{Introduction}

Nowadays researchers have shown a growing interest in mathematical modeling of the process of cancer progression at different levels. Analysis of cancer survival data and related outcomes are necessary to asses cancer treatment programs. Chen and Beck (2008), developed a superstatistical model for the progression of metastasis and the corresponding survival statistics of cancer patients. The importance of superstatistics was illustrated over the last few years, both in applied and theoretical aspects. Superstatistics is a branch of statistical physics devoted originally to the study of non-equilibrium complex systems. The concept of superstatistics can be effectively described by a superposition of several statistics, which was first discussed by Beck and Cohen (2003). The stationary distribution of superstatistical systems is obtained by averaging over the fluctuating parameter $\beta$. Then the system distribution can be written as

$$
p(x)=\int_{0}^{\infty} p_{1}(x \mid \beta) p_{2}(\beta) \mathrm{d} \beta .
$$

Of course this is similar to compound or mixture models in Statistics and for details we can refer to Johnson et al. $(1992,1994)$. A Bayesian method to analyze cancer survival data using Weibull model was discussed by Abrams and Ashby (1996). Molenberghs and Verbeke (2011) developed a Weibull-gamma density for modeling the survival data from cancer patients. 
The basic characteristics of complex systems include long-range correlations, multifractality and non-Gaussian distributions with asymptotic power law behavior. Such systems are not well described by ideas based on the usual statistical mechanics. The $q$-type distributions are effectively used for describing the behavior of complex systems. Recently various authors have introduced several $q$-type distributions such as $q$-exponential, $q$-Weibull, $q$-gamma etc. Such types of distributions have lots of applications in various contexts such as empirical study of stock markets (Politi and Scalas (2008)), train delays (Briggs and Beck (2007)), DNA sequences (Keylock (2005)) etc. A brief review of $q$-distributions in complex systems is give in Picoli et al. (2009). The aim of the present paper is to extend the Weibull-gamma model in the light of $q$-Weibull distribution. The connection between $q$-type distributions including $q$-Weibull model and various entropy measure in statistical literature was studied in detail by Mathai and Haubold (2007).

The organization of the paper is as follows: In section 2, we present the $q$-Weibull distribution and some of its properties. The new generalization of Weibull-gamma model is derived and its structural properties are discussed in section 3. The method of moments for estimating the parameters of the proposed model is studied in section 4 . Section 5 contains the fitting of the new model to Lung cancer survival data.

\section{2. q-Weibull density}

Before going to the discussion of the $q$-Weibull density, we give a connection of this density to Tsallis statistics and pathway idea of Mathai (2005). The connection is based on the fact that the $q$-exponential can be viewed as Tsallis statistics. The $q$-exponential density is obtained when the Tsallis entropy

$$
T_{k, q}=\frac{\sum_{i=1}^{k} p_{i}^{q}-1}{1-q}, q \neq 1
$$

and for the continuous case

$$
T_{k, q}=\frac{\int_{-\infty}^{\infty}[f(x)]^{q} \mathrm{~d} x-1}{1-q}, q \neq 1,
$$

is maximized subject to the conditions that $f$ is a density or probability function and that the first moment is fixed. Then going through an escort density we end up with Tsallis statistics. Here $p_{i}$ is the probability of the $i^{t h}$ state and $q$ is a real parameter. The pathway model and from there the $q$-Weibull, are obtained by optimizing Mathai's entropy, see Mathai and Haubold (2007). The importance of $q$-Weibull density in reliability theory is shown in detail in Assis et al. (2013). The density function of the $q$-Weibull model is

$$
f_{1}(x \mid \theta)= \begin{cases}\beta \theta(2-q) x^{\beta-1}\left[1+\theta(q-1) x^{\beta}\right]^{-\frac{1}{q-1}}, & x \geq 0, q<2, \beta>0, \\ 0, & \text { otherwise }\end{cases}
$$

For $q>1$ we have the form in (2.3). The model in (2.3) consists of three different functional forms. For $q<1$, we can write $\left[1+\theta(q-1) x^{\beta}\right]^{-\frac{1}{q-1}}=\left[1-\theta(1-q) x^{\beta}\right]^{\frac{1}{1-q}}$. The right side remains positive in the finite range $\left[1-\theta(1-q) x^{\beta}\right]>0$ or $0<x<\frac{1}{(\theta(1-q))^{\frac{1}{\beta}}}$. Then for $q<1, f_{1}(x \mid \theta)$ can be 
written as

$$
f_{2}(x \mid \theta)= \begin{cases}\beta \theta(2-q) x^{\beta-1}\left[1-\theta(1-q) x^{\beta}\right]^{\frac{1}{1-q}} 0<x<\frac{1}{(\theta(1-q))^{\frac{1}{\beta}}}, \beta>0 \\ 0, & \text { otherwise. }\end{cases}
$$

When $q \rightarrow 1, f_{1}(x \mid \theta)$ and $f_{2}(x \mid \theta)$ will go to $f_{3}(x \mid \theta)$, where

$$
f_{3}(x \mid \theta)= \begin{cases}\beta \theta x^{\beta-1} \mathrm{e}^{-\theta x^{\beta}}, & \theta, \beta>0, x \geq 0, \\ 0, & \text { otherwise },\end{cases}
$$

which is the Weibull density. When $q \rightarrow 1$, (2.3) becomes Weibull density; when $\beta=1$, (2.3) becomes q-exponential model and when $q \rightarrow 1$, (2.3) becomes (2.5) with $\beta=1$, which is the exponential density. The achievement of the $q$-type distributions is that this new extension of the distribution has its ability to exhibit heavy-tails when compared to the classical distributions. Nowadays $q$-Weibull has been applied to many practical problems in physics, for example in the study of fractal kinetics (Brouers and Costa (2006)), dielectric breakdown in oxides (Costa et al. (2006)), relaxation in heterogeneous system (Brouers et al. (2005)), cyclone victims (Picoli et al. (2003)) etc.

\section{Generalized Weibull-gamma model}

Suppose $x$ is a random variable having Weibull density given in (2.5) with parameters $\theta$ and $\beta$ where $\theta$ follows a gamma density with parameters $\alpha$ and $\delta$, having the density function as

$$
f_{4}(\theta)=\frac{1}{\delta^{\alpha} \Gamma(\alpha)} \theta^{\alpha-1} \mathrm{e}^{-\frac{\theta}{\delta}}, \theta>0, \delta>0, \alpha>0
$$

and $f_{4}(\theta)=0$ elsewhere. Then the density of $x$ is given by

$$
\begin{aligned}
h(x) & =\int_{0}^{\infty} f_{3}(x \mid \theta) f_{4}(\theta) \mathrm{d} \theta \\
& =\frac{\beta}{\Gamma(\alpha) \delta^{\alpha}} x^{\beta-1} \int_{0}^{\infty} \theta^{\alpha} \mathrm{e}^{-\theta x^{\beta}-\frac{\theta}{\delta}} \mathrm{d} \theta \\
& =\beta \delta \alpha \frac{x^{\beta-1}}{\left(1+\delta x^{\beta}\right)^{\alpha+1}}, x \geq 0, \delta>0, \beta>0, \alpha>0,
\end{aligned}
$$

and $h(x)=0$ elsewhere. The density in (3.3) is the same as the generalized Burr density, for details of Burr type model see Rodrigues (1977). The Burr type distributions have been used in a variety of statistical modeling, which includes crop prices, operational risk, market price distribution etc. Also note that by substituting $\beta=1$, (3.3) is reduced to the Lomax density (Lomax (1954)). By suitable transformations, the density function (3.3) can be reduced to some special cases of the beta and $F$ distributions. Tadikamalla (1980) discussed the relationship between the Burr distributions and the various other distributions namely, the Lomax and the Kappa family of distributions. For $\alpha=1, \delta=\frac{\gamma}{\eta}$, the density function (3.3) reduces to

$$
h(x)=\beta \gamma \eta \frac{x^{\beta-1}}{\left(\eta+\gamma x^{\beta}\right)^{2}}, x \geq 0, \beta>0, \gamma>0, \eta>0,
$$

and $h(x)=0$ elsewhere. This is the log-logistic density given in Bennet (1983) and Collett (2003). 


\subsection{Pathway extended gamma model}

Observe that the integrand in (3.2) consists of two factors $\mathrm{e}^{-\theta x^{\beta}}$ and $\theta^{\alpha} \mathrm{e}^{-\frac{\theta}{\delta}}$. The general forms of these can be obtained as the limiting forms of binomial functions. That is

$$
\lim _{p \rightarrow 1} \theta^{r}\left[1+x^{\delta} \theta^{\varepsilon}(p-1)\right]^{-\frac{1}{p-1}}=\theta^{r} \mathrm{e}^{-x^{\delta} \theta^{\varepsilon}},
$$

and

$$
\lim _{q \rightarrow 1} \theta^{\alpha}\left[1+(q-1) \frac{\theta^{\varepsilon}}{\delta}\right]^{-\frac{1}{q-1}}=\theta^{\alpha} \mathrm{e}^{-\frac{\theta^{\varepsilon}}{\delta}}
$$

Thus, the pathway extension of $\theta^{r} \mathrm{e}^{-x^{\delta} \theta^{\varepsilon}}$ and $\theta^{\alpha} \mathrm{e}^{-\frac{\theta^{\varepsilon}}{\delta}}$ are $\theta^{r}\left[1+(p-1) x^{\delta} \theta^{\varepsilon}\right]^{-\frac{1}{p-1}}$ and $\theta^{\alpha}[1+$ $\left.(q-1) \frac{\theta^{\varepsilon}}{\delta}\right]^{-\frac{1}{q-1}}$ respectively. Hence, we may replace the gamma density forms by the corresponding binomial factors. The analysis can be done exactly as described below. In order to avoid complicated expressions we will consider only very special cases of (3.5) and (3.6). Note that if $r=\varepsilon-1$ in (3.5) and $\alpha=\varepsilon-1$ in (3.6) then the analysis is simplified and we will end up with extended Weibull type distributions. This case will be considered in detail below.

We extend the density in (3.3) by using $q$-Weibull density. Then the density function of $x$ is given by $h_{1}(x)$ which has the following form

$$
h_{1}(x)=\frac{\beta(2-q)}{\delta^{\alpha} \Gamma(\alpha)} x^{\beta-1} \int_{0}^{\infty} \theta^{\alpha}\left[1+(q-1) \theta x^{\beta}\right]^{\frac{-1}{q-1}} \mathrm{e}^{-\frac{\theta}{\delta}} \mathrm{d} \theta
$$

for $1<q<2, \alpha, \beta, \delta>0, x \geq 0$. The integral part in (3.7) can be evaluated by using the Mellin convolution property, by taking it as a statistical distribution problem. Let $x_{1}$ and $x_{2}$ be independently distributed real scalar positive random variables. Let their densities be $g_{1}\left(x_{1}\right)$ and $g_{2}\left(x_{2}\right)$ respectively. Consider the transformation $u=\frac{x_{1}}{x_{2}}$ and $v=x_{2}$. Then the density function of $u$ is given by

$$
g(u)=\int_{v} v g_{1}(u v) g_{2}(v) \mathrm{d} v .
$$

Let

$$
g_{1}\left(x_{1}\right)=\mathrm{c}_{1}\left[1+(q-1) x_{1}\right]^{-\frac{1}{q-1}}, x_{1} \geq 0 \text { and } g_{2}\left(x_{2}\right)=\mathrm{c}_{2} x_{2}^{\alpha-1} \mathrm{e}^{-b x_{2}}, x_{2} \geq 0, b>0,
$$

so that $g_{1}$ and $g_{2}$ can create statistical densities for appropriate values of the normalizing constants $c_{1}$ and $c_{2}$. Note that we could have $g_{1}$ and $g_{2}$ in terms of the extended form in (3.5) and (3.6). The procedure described below would have remained the same. Then

$$
c_{1} c_{2} \int_{\theta=0}^{\infty} \theta\left[1+(q-1) \theta x^{\beta}\right]^{-\frac{1}{q-1}} \theta^{\alpha-1} \mathrm{e}^{-\frac{\theta}{\delta}} \mathrm{d} \boldsymbol{\theta}=\int_{\theta=0}^{\infty} \theta g_{1}\left(x^{\beta} \theta\right) g_{2}(\theta) \mathrm{d} \theta, u=x^{\beta} .
$$

Hence the Mellin transform of the left side is the product of the Mellin transforms of the right side. Writing in terms of statistical expectations,

$$
E\left(x_{1}^{s-1}\right)=c_{1} \frac{1}{(q-1)^{s}} \frac{\Gamma(s) \Gamma\left(\frac{1}{q-1}-s\right)}{\Gamma\left(\frac{1}{q-1}\right)},
$$


for $\Re(s)>0, \Re\left(\frac{1}{q-1}-s\right)>0, q>1$, where $\Re($.$) is the real part of ($.$) , and$

$$
E\left(x_{2}{ }^{1-s}\right)=c_{2} \frac{1}{(b)^{\alpha-s+1}} \Gamma(\alpha-s+1), \Re(\alpha-s+1)>0 .
$$

From (3.10) and (3.11),

$$
E\left(u^{s-1}\right)=c_{1} c_{2} \frac{1}{(q-1)^{s} b^{(\alpha-s+1)}} \frac{\Gamma(s) \Gamma\left(\frac{1}{q-1}-s\right) \Gamma(\alpha-s+1)}{\Gamma\left(\frac{1}{q-1}\right)}
$$

for $b>0, q>1, \Re(s)>0, \Re\left(\frac{1}{q-1}-s\right)>0$ and $\Re(\alpha-s+1)>0$. Then the integral is available by taking the inverse Mellin transform and it is the following:

$$
\begin{aligned}
& c_{1} c_{2} \int_{\theta=0}^{\infty} \theta\left[1+(q-1) \theta x^{\beta}\right]^{-\frac{1}{q-1}} \theta^{\alpha-1} \mathrm{e}^{-\frac{\theta}{\delta}} \mathrm{d} \theta \\
& =c_{1} c_{2} \frac{\delta^{\alpha+1}}{\Gamma\left(\frac{1}{q-1}\right)} \frac{1}{2 \pi i} \int_{L} \Gamma(s) \Gamma\left(\frac{1}{q-1}-s\right) \Gamma(\alpha-s+1)\left((q-1) x^{\beta} \delta\right)^{-s} \mathrm{~d} s \\
& =c_{1} c_{2} \frac{\delta^{\alpha+1}}{\Gamma\left(\frac{1}{q-1}\right)} \mathrm{G}_{2,1}^{1,2}\left[\left.(q-1) x^{\beta} \delta\right|_{0} ^{1-\frac{1}{q-1},-\alpha}\right],
\end{aligned}
$$

for $1<q<2, x>0$, and $\delta>0$, where $\mathrm{G}$ is the G-function. For the definition, theory and applications of G-function, see Mathai (1993). Observe that $c_{2}$ from (3.11) is $\frac{\Gamma(\alpha)}{\alpha}$ and $c_{1}$ from (3.10) is available by putting $s=1$ and using the fact that $E(1)=1$. Then substituting $c_{1}$ and $c_{2}$ in (3.12) and inverting (3.12) we have,

$$
h_{1}(x)=\frac{\beta(2-q) \delta}{\Gamma(\alpha) \Gamma\left(\frac{1}{q-1}\right)} x^{\beta-1} \mathrm{G}_{2,1}^{1,2}\left[\left.(q-1) x^{\beta} \delta\right|_{0} ^{1-\frac{1}{q-1},-\alpha}\right],
$$

for $x>0, \delta>0,1<q<2, \beta>0$, and $h_{1}(x)=0$ elsewhere.

Now for $q<1$, the density function of $x$ has the following form:

$$
h_{2}(x)=\frac{\beta(2-q)}{\delta^{\alpha} \Gamma(\alpha)} x^{\beta-1} \int_{0}^{\frac{1}{(\theta(1-q))^{\frac{1}{\beta}}}} \theta^{\alpha}\left[1-\theta(1-q) x^{\beta}\right]^{\frac{1}{1-q}} \mathrm{e}^{-\frac{\theta}{\delta}} \mathrm{d} \theta .
$$

For convenience of integration, let us assume that $d=\frac{1}{(\theta(1-q))^{\frac{1}{\beta}}}$. For $q<1$, let

$$
I_{1}=\int_{0}^{d} \theta^{\alpha}\left[1-\theta(1-q) x^{\beta}\right]^{\frac{1}{1-q}} \mathrm{e}^{-\frac{\theta}{\delta}} \mathrm{d} \theta
$$

where $d=\frac{1}{(\theta(1-q))^{\frac{1}{\beta}}}$ and $\beta>0$. Then $I_{1}$ is the product of the two integrable functions. Hence we can apply Mellin convolution property for finding the value of the integral. Let

$$
g_{3}\left(x_{1}\right)=c_{3}\left[1-(1-q) x_{1}\right]^{\frac{1}{1-q}},\left[1-(1-q) x_{1}\right] \geq 0 \text { and } g_{4}\left(x_{2}\right)=c_{4} x_{2}{ }^{\alpha-1} \mathrm{e}^{-b x_{2}}, x_{2} \geq 0, b>0,
$$

where $c_{3}$ and $c_{4}$ are normalizing constants. Then proceeding as in the case for $q>1$, we have

$$
h_{2}(x)=\frac{\delta \beta(2-q) \Gamma\left(\frac{1}{1-q}+1\right)}{\Gamma(\alpha)} x^{\beta-1} \mathrm{G}_{2,1}^{1,1}\left[\left.(1-q) \delta x^{\beta}\right|_{0} ^{-\alpha, 1+\frac{1}{1-q}}\right],
$$


for $x>0, \delta>0, \beta>0, \alpha>0, q<1$ and $h_{2}(x)=0$ elsewhere.

Theorem 3.1. When the conditional density of $x \mid \theta$ is of the form $f_{1}(x \mid \theta)$ in (2.3), and the marginal density of $\theta$ is of the form of $f_{4}(\theta)$ in (3.1), then for $q>1$, the unconditional density of $x$ denoted by $h_{1}(x)$, is given by

$$
h_{1}(x)=\frac{\beta(2-q) \delta}{\Gamma(\alpha) \Gamma\left(\frac{1}{q-1}\right)} x^{\beta-1} \mathrm{G}_{2,1}^{1,2}\left[\left.(q-1) x^{\beta} \delta\right|_{0} ^{1-\frac{1}{q-1},-\alpha}\right],
$$

for $x>0, \delta>0,1<q<2, \beta>0$, and $h_{1}(x)=0$ elsewhere.

For $q<1$

$$
h_{2}(x)=\frac{\delta \beta(2-q) \Gamma\left(\frac{1}{1-q}+1\right)}{\Gamma(\alpha)} x^{\beta-1} \mathrm{G}_{2,1}^{1,1}\left[\left.(1-q) \delta x^{\beta}\right|_{0} ^{-\alpha, 1+\frac{1}{1-q}}\right],
$$

for $x>0, \delta>0, \beta>0, \alpha>0$ and $h_{2}(x)=0$ elsewhere. As $q \rightarrow 1, h_{1}(x)$ and $h_{2}(x)$ tend to $h(x)$ where $h(x)$ is the usual Weibull-gamma model with the parameters $\beta, \delta$ and $\alpha$, and it is given by

$$
h(x)=\beta \delta \alpha \frac{x^{\beta-1}}{\left(1+\delta x^{\beta}\right)^{\alpha+1}},
$$

for $x \geq 0, \beta, \delta, \alpha>0$ and $h(x)=0$ elsewhere.

\section{Special Cases}

Many compound distributions are special cases of the extended Weibull-gamma model introduced in section 2. Some of them are given below.

(1) Rayleigh-gamma density is obtained from (3.18), by letting $q \rightarrow 1$ and $\beta=2$.

(2) The extended Rayleigh-gamma model is obtained from (3.18), by letting $\beta=2$.

(3) The exponential-gamma density is obtained from (3.18), by letting $q \rightarrow 1$ and $\beta=1$.

(4) The extended exponential-gamma density is obtained from (3.18), by letting $\beta=1$.

The behavior of the density functions is given in the following figures for selected values of the parameters.

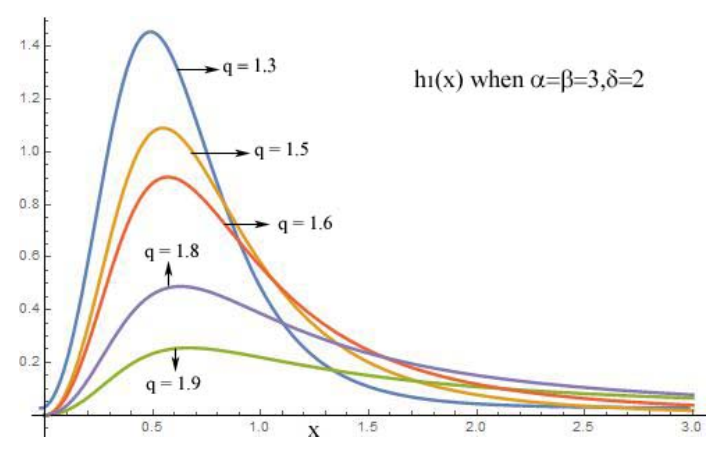

Figure 1 (a)

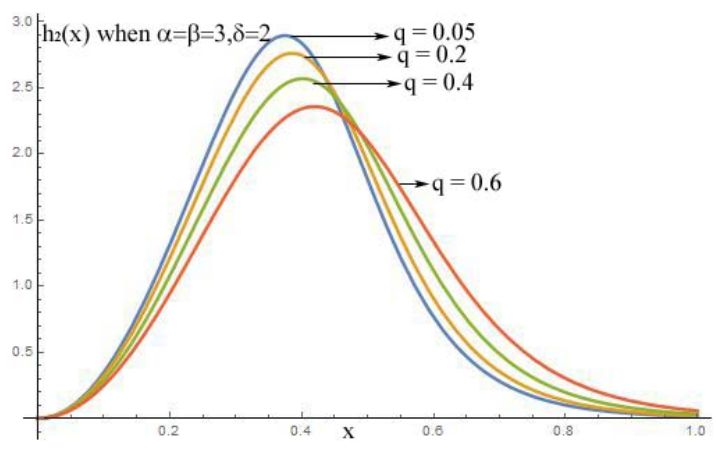

Figure $1(b)$

Density functions of extended Weibull-gamma distribution. 


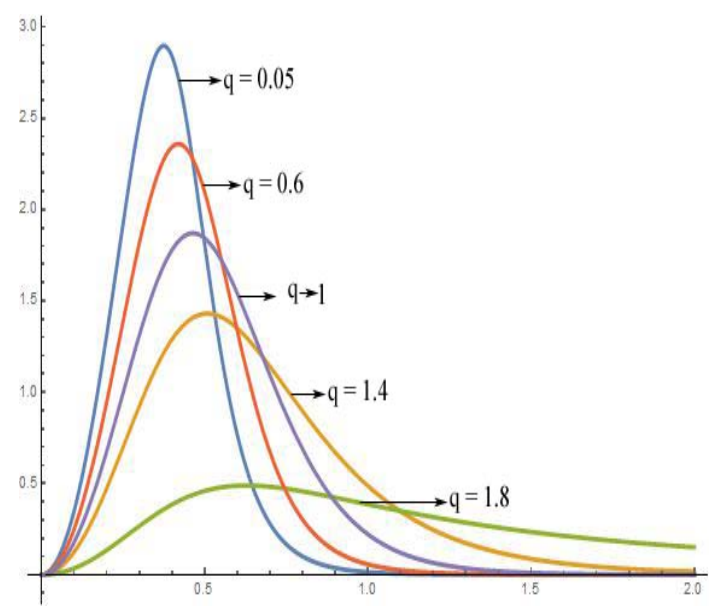

Figure 2

Comparison between Weibull-gamma $(q \rightarrow 1)$ and for different values of $q$.

The effect of the parameters can be easily seen from these graphs. Figure $1(a)$ and $1(b)$ illustrate the extended Weibull-gamma model when the parameters $\alpha, \beta, \delta$ are fixed and $q$ is changing. From these figures, we can say that the density function has thicker tail for $q<1$ than $q>1$. Figure 2 shows a comparison between Weibull-gamma model $(q \rightarrow 1)$ and extended Weibull-gamma model for different values of $q$.

\section{Properties}

This section discusses the statistical properties for the extended Weibull-gamma distribution.

The $h^{\text {th }}$ moment of a random variable $x$ with density function given by (3.18). Then the $h^{\text {th }}$ moment of $x$ is

$$
\begin{aligned}
E\left(x^{h}\right) & =\int_{0}^{\infty} x^{h} h_{1}(x) \mathrm{d} x \\
& =\frac{(2-q) \delta \Gamma\left(\frac{h}{\beta}+1\right) \Gamma\left(\frac{1}{q-1}-\frac{h}{\beta}-1\right) \Gamma\left(\alpha-\frac{h}{\beta}\right)}{\Gamma(\alpha) \Gamma\left(\frac{1}{q-1}\right)((q-1) \delta)^{\frac{h}{\beta}+1}},
\end{aligned}
$$

for $\alpha>0,1<q<2, \Re\left(\frac{h}{\beta}\right)>0, \Re\left(\alpha-\frac{h}{\beta}\right)>0$, and $\Re\left(\frac{1}{q-1}-\frac{h}{\beta}-1\right)>0$.

The mean value is obtained when $h=1$. That is

$$
E(x)=\frac{(2-q) \delta \Gamma\left(\frac{1}{\beta}+1\right) \Gamma\left(\frac{1}{q-1}-\frac{1}{\beta}-1\right) \Gamma\left(\alpha-\frac{1}{\beta}\right)}{\Gamma(\alpha) \Gamma\left(\frac{1}{q-1}\right)(\delta(q-1))^{\frac{1}{\beta}+1}}
$$

for $\alpha>0,1<q<2, \alpha-\frac{1}{\beta}>0, \frac{1}{q-1}-\frac{1}{\beta}-1>0, \beta, \delta>0$.

$E\left(x^{2}\right)$ is obtained when $h=2$ and the variance of $x$ is $\operatorname{Var}(x)=E\left(x-(E(x))^{2}\right)$ is

$$
\operatorname{Var}(x)=\frac{(2-q) \delta}{\Gamma(\alpha) \Gamma\left(\frac{1}{q-1}\right)(\delta(q-1))^{\frac{2}{\beta}+1}} \times
$$




$$
\left[\Gamma\left(\frac{2}{\beta}+1\right) \Gamma\left(\frac{1}{q-1}-\frac{2}{\beta}-1\right) \Gamma\left(\alpha-\frac{2}{\beta}\right)-\frac{(2-q)\left(\Gamma\left(\frac{1}{\beta}+1\right)\right)^{2}\left(\Gamma\left(\frac{1}{q-1}-\frac{1}{\beta}-1\right)\right)^{2}\left(\Gamma\left(\alpha-\frac{1}{\beta}\right)\right)^{2}}{\Gamma(\alpha) \Gamma\left(\frac{1}{q-1}\right)(q-1)}\right]
$$

for $\alpha-\frac{1}{\beta}>0,1<q<2, \alpha-\frac{2}{\beta}>0, \frac{1}{q-1}-\frac{1}{\beta}-1>0, \frac{1}{q-1}-\frac{2}{\beta}-1>0, \alpha>0, \beta, \delta>0$.

For $q<1$ the $h^{\text {th }}$ moment of $x$ becomes

$$
E\left(x^{h}\right)=\frac{(2-q) \delta \Gamma\left(\frac{1}{1-q}+1\right) \Gamma\left(\frac{h}{\beta}+1\right) \Gamma\left(\alpha-\frac{h}{\beta}\right)}{\Gamma(\alpha)((1-q) \delta)^{\frac{h}{\beta}+1} \Gamma\left(2+\frac{1}{1-q}+\frac{h}{\beta}\right)},
$$

for $\alpha>0, \Re\left(\alpha-\frac{h}{\beta}\right)>0, \Re\left(\frac{1}{1-q}+\frac{h}{\beta}\right)>0, \delta>0, \beta>0$.

The corresponding mean and variance are obtained similarly for the above case.

\section{Laplace Transform}

The Laplace transform $L_{h_{1}}(t)$ of the density function $h_{1}(x)$ is given by

$$
L_{h_{1}}(t)=\int_{0}^{\infty} \mathrm{e}^{-t x} h_{1}(x) \mathrm{d} x .
$$

Using the equation (2.19) in Mathai et al. (2010), we get

$$
L_{h_{1}}(t)=\frac{\beta \delta(2-q)}{\Gamma(\alpha) \Gamma\left(\frac{1}{q-1}\right) t^{\beta}} \mathrm{H}_{1,3}^{3,1}\left[\left.\frac{t^{\beta}}{(q-1) \delta}\right|_{(\beta, \beta),\left(\frac{1}{q-1}, 1\right),(1+\alpha, 1)} ^{(1,1)}\right],
$$

for $t>0, \beta>0, \delta>0, \alpha>0,1<q<2$, where $\mathrm{H}$ (.) is the H-function, for details see Mathai et al. (2010). For $q<1$, the Laplace transform is given by

$$
L_{h_{2}}(t)=\frac{\beta \delta(2-q) \Gamma\left(\frac{1}{1-q}+1\right)}{\Gamma(\alpha) t^{\beta}} \mathrm{H}_{1,3}^{2,1}\left[\left.\frac{t^{\beta}}{(1-q) \delta}\right|_{(\beta, \beta),\left(-\frac{1}{1-q}, 1\right),(1+\alpha, 1)} ^{(1,1)}\right]
$$

for $t>0, \beta>0, \delta>0, \alpha>0$.

\section{Distribution Function}

The distribution function $H_{1}(x)$ of the extended Weibull-gamma random variable $x$ is given by the following:

For $q>1$,

$$
H_{1}(x)=1-\frac{(2-q)}{\Gamma(\alpha) \Gamma\left(\frac{1}{q-1}\right)(q-1)} \mathrm{H}_{3,2}^{2,2}\left[\left.(\delta(q-1))^{\frac{1}{\beta}} x\right|_{(0,1),\left(1, \frac{1}{\beta}\right)} ^{\left(2-\frac{1}{q-1}, \frac{1}{\beta}\right),\left(1-\alpha, \frac{1}{\beta}\right),(1,1)}\right],
$$

for $x>0, \alpha>0, \beta>0,1<q<2$.

When $q<1$, the distribution function $H_{2}(x)$ is given by

$$
H_{2}(x)=1-\frac{(2-q) \Gamma\left(\frac{1}{1-q}+1\right)}{\Gamma(\alpha)(1-q)} \mathrm{H}_{3,2}^{2,1}\left[\left.(\delta(1-q))^{\frac{1}{\beta}} x\right|_{(0,1),\left(1, \frac{1}{\beta}\right)} ^{\left(1-\alpha, \frac{1}{\beta}\right),\left(2+\frac{1}{1-q}, \frac{1}{\beta}\right),(1,1)}\right],
$$

for $x>0, \alpha>0, \beta>0$. 
The behavior of the distribution functions $H_{1}(x)$ and $H_{2}(x)$ for the different values of the parameters can be seen from the following graphs.

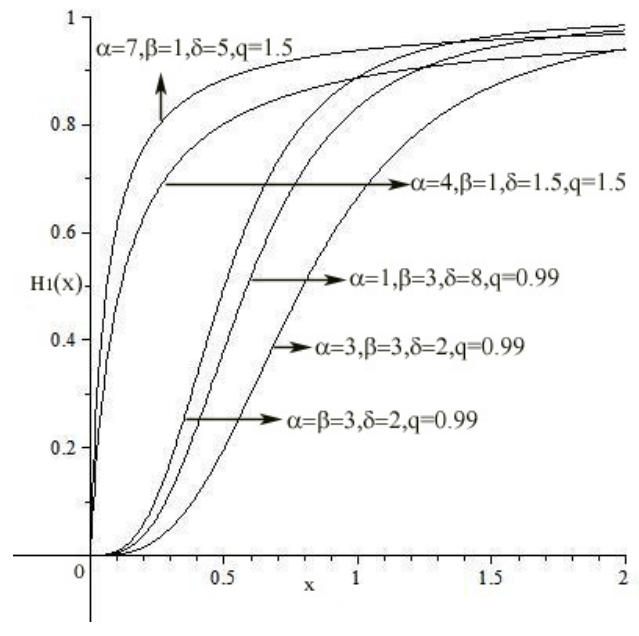

Figure 4

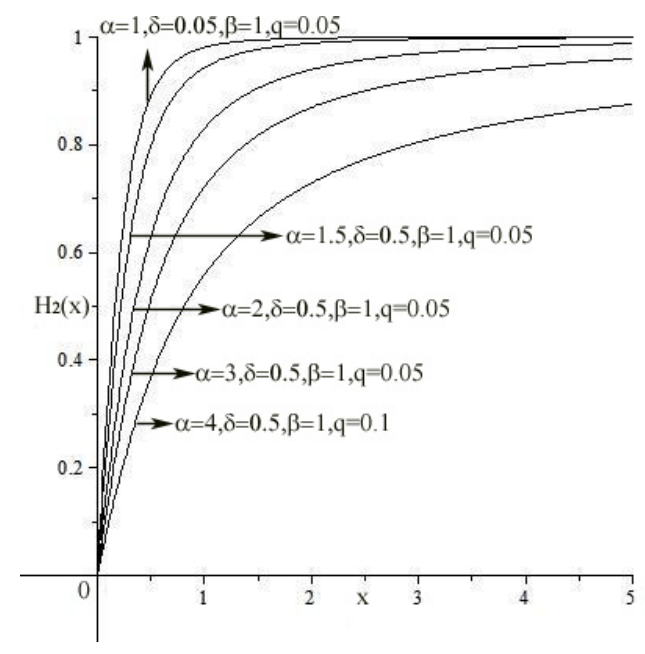

Figure 5

Figure 4. The graph of $H_{1}(x)$ for various values of the parameters $\alpha, \beta, \delta$ and $q$. Figure 5. The graph of $H_{2}(x)$ for various values of the parameters $\alpha, \beta, \delta$ and $q$.

\section{Reliability and Hazard Function}

Here we discuss the behavior of hazard rate function on the family of extended Weibull-gamma model. The reliability function $R(t)=1-F(t)$ of the extended Weibull-Gamma model is given by

$$
R_{1}(t)=\frac{(2-q)}{\Gamma(\alpha) \Gamma\left(\frac{1}{q-1}\right)(q-1)} \mathrm{H}_{3,2}^{2,2}\left[\left.(\delta(q-1))^{\frac{1}{\beta}} t\right|_{(0,1),\left(1, \frac{1}{\beta}\right)} ^{\left(1-\alpha, \frac{1}{\beta}\right),\left(2-\frac{1}{q-1}, \frac{1}{\beta}\right),(1,1)}\right]
$$

for $t>0, \alpha>0, \beta>0,1<q<2$.

The hazard rate function $\lambda_{1}(t)=\frac{f(t)}{R(t)}$ of the extended Weibull-gamma random variable is

$$
\lambda_{1}(t)=(\delta(q-1))^{\frac{1}{\beta}} \frac{t^{\beta-1} \mathrm{H}_{2,1}^{1,2}\left[\left.(\delta(q-1))^{\frac{1}{\beta}} t\right|_{\left(1-\frac{1}{\beta}, \frac{1}{\beta}\right)} ^{\left(1-\alpha-\frac{1}{\beta}, \frac{1}{\beta}\right),\left(2-\frac{1}{q-1}-\frac{1}{\beta}, \frac{1}{\beta}\right)}\right]}{\mathrm{H}_{3,2}^{2,2}\left[\left.(\delta(q-1))^{\frac{1}{\beta}} t\right|_{(0,1),\left(1, \frac{1}{\beta}\right)} ^{\left(1-\alpha, \frac{1}{\beta}\right),\left(2-\frac{1}{q-1}, \frac{1}{\beta}\right),(1,1)}\right]},
$$

for $t>0, \delta>0$. The expression of $\lambda_{1}(t)$ is complicated, but for particular values of the parameters $\alpha, \beta, \delta$ and $q$, the hazard rate function can be the ratio of Meijer G-function. Meijer G-function can be evaluated using the softwares Maple 14 or Mathematica.

The graphs of $\lambda_{1}(t)$ for various values of the parameters are given in the following figures. 


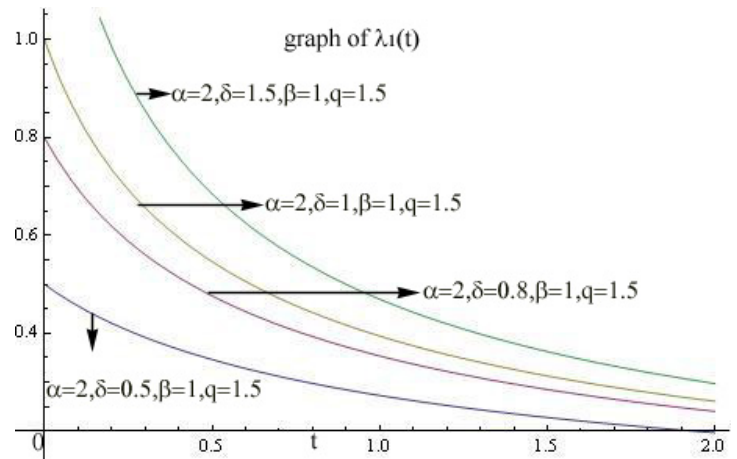

Figure 6

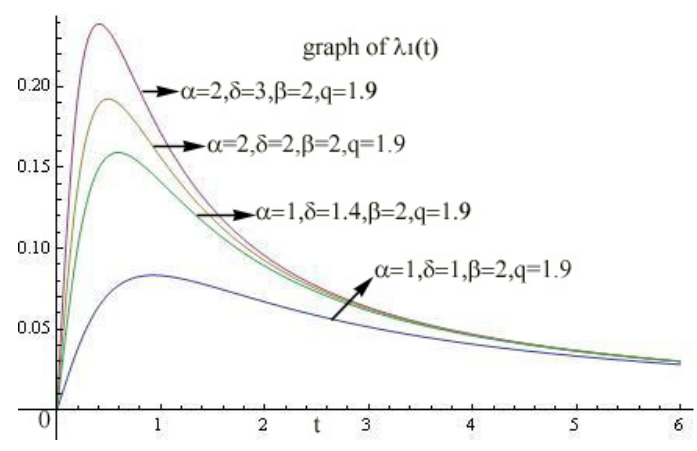

Figure 7

Figure 6. The graph of $\lambda_{1}(t)$ for various values of the parameters $\alpha, \beta, \delta$ and $q$. Figure 7. The graph of $\lambda_{1}(x)$ for various values of the parameters $\alpha, \beta, \delta$ and $q$. For $1<q<2, \beta=1$, the failure rate of extended Weibull-gamma model is monotonically decreasing function and when $1<q<2, \beta=2$, the hazard rate function is unimodal type.

For $q<1$, the reliability and hazard rate functions are given by

$$
R_{2}(t)=\frac{(2-q) \Gamma\left(\frac{1}{1-q}+1\right)}{\Gamma(\alpha)(1-q)} \mathrm{H}_{3,2}^{2,1}\left[\left.(\delta(1-q))^{\frac{1}{\beta}} t\right|_{(0,1),\left(1, \frac{1}{\beta}\right)} ^{\left(1-\alpha, \frac{1}{\beta}\right),\left(2+\frac{1}{1-q}, \frac{1}{\beta}\right),(1,1)}\right],
$$

for $\alpha>0, x>0$, and hazard function

$$
\lambda_{2}(t)=(\delta(1-q))^{\frac{1}{\beta}} \frac{t^{\beta-1} \mathrm{H}_{2,1}^{1,1}\left[\left.(\delta(1-q))^{\frac{1}{\beta}} t\right|_{(0,1),\left(1-\frac{1}{\beta}, \frac{1}{\beta}\right)} ^{\left(1-\alpha-\frac{1}{\beta}, \frac{1}{\beta}\right),\left(2+\frac{1}{1-q}-\frac{1}{\beta}, \frac{1}{\beta}\right),(1,1)}\right]}{\mathrm{H}_{3,2}^{2,1}\left[\left.(\delta(1-q))^{\frac{1}{\beta}} t\right|_{(0,1),\left(1, \frac{1}{\beta}\right)} ^{\left(1-\alpha, \frac{1}{\beta}\right),\left(2+\frac{1}{1-q}, \frac{1}{\beta}\right),(1,1)}\right]},
$$

for $t>0, \delta>0, \beta>0$.

The graphs of $\lambda_{2}(t)$ for various values of the parameters are given in the following figures.

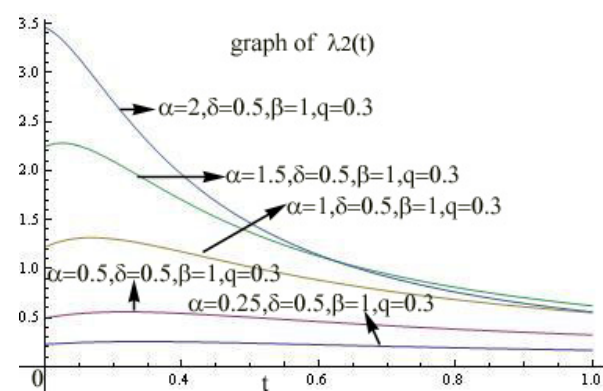

Figure 8

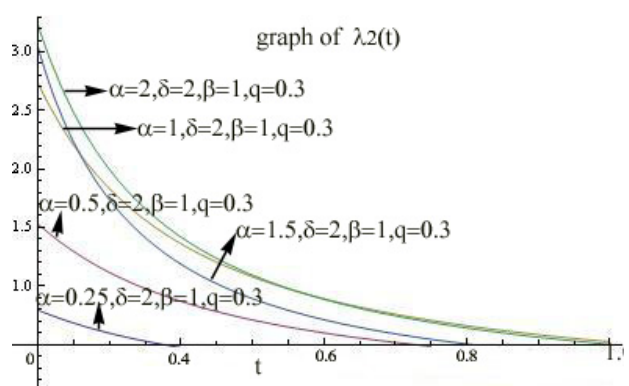

Figure 9

Figure 8. The graph of $\lambda_{2}(t)$ for various values of the parameters $\alpha, \beta, \delta$ and $q$. Figure 9. The graph of $\lambda_{2}(x)$ for various values of the parameters $\alpha, \beta, \delta$ and $q$. For $q<1$, the extended Weibull-gamma failure rate is monotonically decreasing function, which is shown in Figure 8 and Figure 9. 
The hazard rate function of the extended Weibull-gamma model has shape like log-logistic, generalized Weibull and log-normal distributions. Which shows the importance of the new model in the area of reliability and survival analysis.

\section{Estimation of parameters}

Before a density function can be used to model any given data the important factor is to estimate the parameters in the model. Here we use method of moments for estimating the parameters of the extended Weibull-gamma model. The method of moments consists of equating sample moments with corresponding population moments and then the parameters are estimated by solving these equations.

Let $m_{j}$ be the $j^{t h}$ sample moment. In (4.2), put $h=1,2,3$, we get the first three moments of the extended Weibull-gamma model and then we equate the sample moments with the corresponding population moments. That is,

$$
m_{1}=\hat{E}(x)=\frac{(2-\hat{q}) \hat{\delta} \Gamma\left(\frac{1}{\hat{\beta}}+1\right) \Gamma\left(\frac{1}{\hat{q}-1}-\frac{1}{\hat{\beta}}-1\right) \Gamma\left(\hat{\alpha}-\frac{1}{\hat{\beta}}\right)}{\Gamma(\hat{\alpha}) \Gamma\left(\frac{1}{\hat{q}-1}\right)((\hat{q}-1) \hat{\delta})^{\frac{1}{\hat{\beta}}}+1},
$$

for $\hat{\alpha}>0, \hat{\alpha}-\frac{1}{\hat{\beta}}>0, \frac{1}{\hat{q}-1}-\frac{1}{\hat{\beta}}>0$, where a hat indicates the estimated value.

$$
m_{2}=\hat{E}\left(x^{2}\right)=\frac{(2-\hat{q}) \hat{\delta} \Gamma\left(\frac{2}{\hat{\beta}}+1\right) \Gamma\left(\frac{1}{\hat{q}-1}-\frac{2}{\hat{\beta}}-1\right) \Gamma\left(\hat{\alpha}-\frac{2}{\hat{\beta}}\right)}{\Gamma(\hat{\alpha}) \Gamma\left(\frac{1}{\hat{q}-1}\right)((\hat{q}-1) \hat{\delta})^{\frac{2}{\hat{\beta}}}+1},
$$

for $\hat{\alpha}>0, \hat{\alpha}-\frac{2}{\hat{\beta}}>0, \frac{1}{\hat{q}-1}-\frac{2}{\hat{\beta}}>0$.

$$
m_{3}=\hat{E}\left(x^{3}\right)=\frac{(2-\hat{q}) \hat{\delta} \Gamma\left(\frac{3}{\hat{\beta}}+1\right) \Gamma\left(\frac{1}{\hat{q}-1}-\frac{3}{\hat{\beta}}-1\right) \Gamma\left(\hat{\alpha}-\frac{3}{\hat{\beta}}\right)}{\Gamma(\hat{\alpha}) \Gamma\left(\frac{1}{\hat{q}-1}\right)((\hat{q}-1) \hat{\delta})^{\frac{3}{\hat{\beta}}}+1},
$$

for $\hat{\alpha}>0, \hat{\alpha}-\frac{3}{\hat{\beta}}>0, \frac{1}{\hat{q}-1}-\frac{3}{\hat{\beta}}>0$.

The parameter $\delta$ can be removed using the ratio of moments. Suppose we take the ratio $\frac{m_{2}{ }^{2}}{m_{1}{ }^{2}}$ and $\frac{m_{1} m_{3}}{m_{2}{ }^{2}}$. Thus we see that $\frac{m_{2}}{m_{1}{ }^{2}}$ and $\frac{m_{1} m_{3}}{m_{2}{ }^{2}}$ are equations in $\hat{q}, \hat{\alpha}$ and $\hat{\beta}$ in terms of gamma functions. The estimators of the parameters can be evaluated as follows:

Case. 1: $\alpha$ is fixed:

If the parameter $\alpha$ is fixed, then we have two equations in terms of $\hat{\beta}$ and $\hat{q}$. Due to the presence of gamma functions in the moment equations $\frac{m_{2}}{m_{1}{ }^{2}}$ and $\frac{m_{1} m_{3}}{m_{2}{ }^{2}}$, the explicit solution for the parameters is difficult. But with the help of Newton-Raphson iteration method, we can estimate the parameters.

Case. 2: $\beta$ is fixed:

In this case, the moment equations are in terms of $\hat{q}$ and $\hat{\alpha}$. Using the above procedure we can estimate the parameters $q$ and $\alpha$.

Case. 3: When all the parameters are unknown:

Let $m_{1}, m_{2}$ and $m_{3}$ be the first three sample moments and let $E(x), E\left(x^{2}\right), E\left(x^{3}\right)$ be the corresponding population moments. The parameters are estimated by using the moment equations $\frac{m_{2}}{m_{1}{ }^{2}}$ and $\frac{m_{1} m_{3}}{m_{2}{ }^{2}}$. Thus we see that $\frac{m_{2}}{m_{1}{ }^{2}}$ and $\frac{m_{1} m_{3}}{m_{2}{ }^{2}}$ are equations in $\hat{q}, \hat{\alpha}$ and $\hat{\beta}$ in terms of gamma functions. For the range of values of $q, \alpha$ and $\beta$, the gamma is well defined and we can solve for the parameters 
by using $\frac{m_{2}}{m_{1}{ }^{2}}$ and $\frac{m_{1} m_{3}}{m_{2}{ }^{2}}$ with any numerical technique. The moment estimators are not easy to evaluate when all the parameters are unknown. If we take the moment of order $h$ which are multiples of $\beta$, then the estimator can be obtained explicitly.

\section{Application}

In this section, the usefulness of the extended Weibull-gamma model is illustrated on a real data set.

The above results are essentially used for modeling and inference of cancer survival data, the methodology is applicable for identification of distribution and estimation of parameters. Here we test the fitting of the proposed model (3.18) which is compared with the Weibull-gamma model (3.3) on lung cancer survival data. Lung cancer is the leading cause of cancer death and the second most diagnosed cancer in both men and women. The lung cancer data is collected from United States cancer statistics for the years 1999 to 2010.

Maple 14 has been used for computing the data moments and estimating the parameters (by method of moments). The adequacy of the model is tested using Kolmogrov-Smirnov (KS) test statistic. The estimation results and Kolmogrov-Smirnov test statistic results are summarized in Table 1.

\begin{tabular}{|c|c|c|}
\hline Distribution & Parameter estimation & KS test \\
\hline Extended Weibull-gamma $(q>1)$ & $\hat{\delta}=0.586, \hat{\alpha}=1, \hat{\beta}=2.34, q=1.1$ & 0.447 \\
\hline Extended Weibull-gamma $(q<1)$ & $\hat{\delta}=1.564, \hat{\alpha}=1, \hat{\beta}=0.237, q=0.5$ & 0.435 \\
\hline Weibull-gamma & $\hat{\delta}=1.568, \hat{\alpha}=1.87, \hat{\beta}=4.56$ & 0.511 \\
\hline
\end{tabular}

Table 1. Parameter estimation and KS test statistic values

The probability plots are given in the following figures.

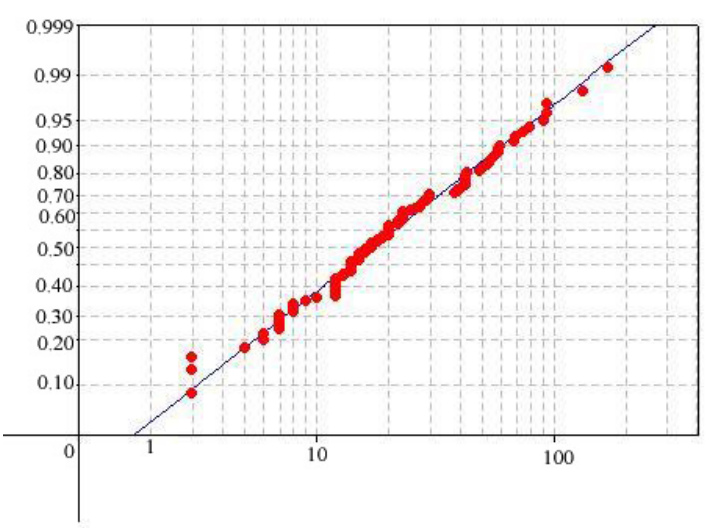

Figure 10

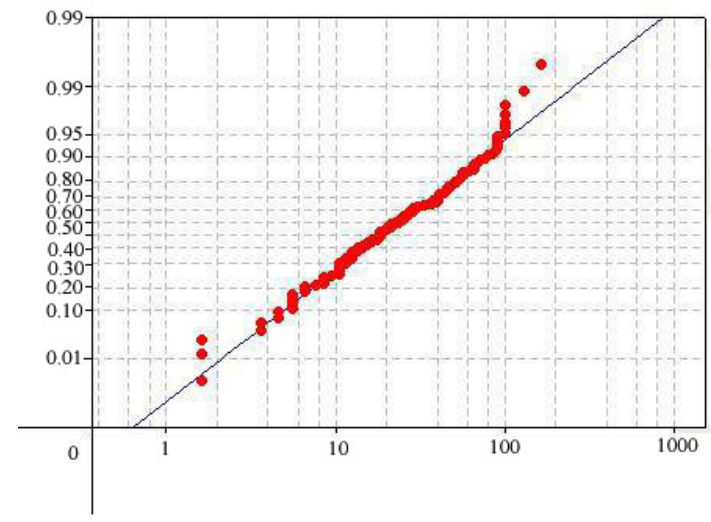

Figure 11

Figure 10. The probability plot for the extended Weibull-gamma model.

Figure 11 . The probability plot for the Weibull-gamma model. 
From the Kolmogrov-smirnov goodness of fits test, we can observe that the extended Weibullgamma model fits fairly well to the lung cancer data. However, the extended Weibull-gamma model produces the lowest Kolmogrov-smirnov test statistic value and therefore fits better than the usual Weibull-gamma model.

\section{Acknowledgements}

The author would like to thank Professor A. M. Mathai of the Centre for Mathematical Sciences for the fruitful discussions. The author would like to thank the Department of Science and Technology and UGC Government of India, New Delhi, for the financial assistance for this work under projectnumber SR/S4/MS:287/05, and the Centre for Mathematical Sciences for providing all facilities.

\section{References}

[1] L. L. Chen and C. Beck, A superstatistical model of metastasis and cancer survival, Physica A, 387 (2008) 3162-3172.

[2] C. Beck and E. G. D. Cohen, Superstatistcs, Physica A, 322 (2003) 267-275.

[3] N. L. Johnson, S. Kotz and A. Kemp, Univariate Discrete Distributions, $2^{\text {nd }}$ edition, John Wiley, New York, (1992).

[4] N. L. Johnson, S. Kotz and N. Balakrishanan, Continuous Univariate Distributions, $2^{\text {nd }}$ edition, John Wiley, New York, (1994).

[5] K. Abrams, D. Ashby, J. Houghton and D. Riley, Assessing Drug Interactions: Tamoxifien and Cyclophosphamide, Bayesian Biostatistics, Marcel Dekker, (1996) 531-554.

[6] Geert Molenberghs, and Geert Verbeke, On the Weibull-gamma frailty model, its infinite moments, and its connection to generalized log-logistic, logistic, Cauchy, and extreme-value distributions, Journal of Statistical Planning and Inference, 141 (2011) 861-868.

[7] M. Politi and E. Scalas, Fitting the empirical distribution of intertrade durations, Physica A, 387 (2008) 2025-2034.

[8] K. Briggs, C. Beck, Modelling train dealys with q-exponential functions, Physica A, 378 (2007) 498504.

[9] C. J. Keylock, Describing the recurrence interval of extreme floods using nonextensive thermodynamics and Tsallis statistics, Advances in Water Resources, 28 (2005) 773-778.

[10] S. Picoli, R. S. Mendes, L. S. Malacarne and R. P. B. Santos, $q$-distributions in complex systems: a brief review, Brazilian Journal of Physics, 39 (2009) 468-474.

[11] A. M. Mathai and H. J. Haubold, Pathway model, superstatistics Tsallis statistics and a generalized measure of entropy, Physica A, 375 (2007) 110-122.

[12] E. M. Assis, E. P. Borges and S. A. B. Vieira de Melo, Generalized q-Weibull model and the bathtub curve, International Journal of Quality \& Reliability Management, 30 (2013) 720-736.

[13] F. Brouers and O. Sotolongo-Costa, Generalized fractal kinetics in complex systems (application to biophysics and biotechnology), Physica A, 368 (2006) 165-175.

[14] U. M. S. Costa, V. N. Freire, L. C. Malacarne, R. S. Mendes, Jr. S.Picoli, E. E. Vasconcelos and Jr. E. F. Da Silva, An improved description of the dielectric breakdown in oxides based on a generalized Weibull distribution, Physica A, 361 (2006) 209-215.

[15] F. Brouers, O. Sotolongo, F. Marquez and J. P. Pirard, Microporous and heterogeneous surface adsorption isotherms arising from Levy distributions, Physica A, 349 (2005) 271-282.

[16] Jr. S. Picoli, R. S. Mendes and L. C. Malacarne, q-exponential, Weibull, and q-Weibull distributions: an empirical analysis, Physica A, 324 (2003) 678-688.

[17] R. N. Rodriguez, A guide to the Burr type XII distributions, Biometrika, 64 (1977) 129-134.

[18] K. S. Lomax, Business failure: Another example of the analysis of the failure data, Journal of American Statistical Association, 49 (1954) 847-852.

[19] P. R. Tadikamalla, A look at the Burr and related distributions, International statistical Review, 48 (1980) 337-344. 
[20] S. Bennet, Log-logistic regression models for survival data, Applied statistics, 32 (1983) 165-171.

[21] D. Collett, Modeling Survival Data in Medical Research, $2^{\text {nd }}$ edition, CRC press, Boca Raton, (2003).

[22] A. M. Mathai, A Handbook of Generalized Special Functions for Statistical and Physical Sciences, Clarendon Press, Oxford, (1993).

[23] A. M. Mathai, R. K. Saxena and H. J. Haubold, The H-function: Theory and Applications, Springer, New York, (2010). 